\title{
Positive Position Feedback Active Vibration Control of a Smart Cantilever Beam Using ANSYS
}

\author{
A.A. El Badawy \\ Mechatronics Department, German University in Cairo, Cairo, \\ Egypt and Mechanical Engineering Department \\ Al-Azhar University \\ Cairo, Egypt
}

\author{
M.M. El Masry \\ Mechatronics Department \\ German University in Cairo \\ Cairo, Egypt
}

\begin{abstract}
In this work, the active vibration control of a uniform cantilever beam using piezoelectric materials subjected to transverse vibrations is studied. The equation of motion of a beam bonded with the piezoelectric actuator is realized based on the Euler Bernoulli beam theory and the Hamilton's principle. A linear time invariant state space model is derived. Numerical simulations of the equation of motion are performed. Moreover, a finite element model of the beam-piezo system is done using ANSYS APDL $@$. Two control algorithms were also implemented using ANSYS APDL code to reduce the flapwise bending vibrations of the beam. The two control techniques are: Linear quadratic regulator (LQR) and positive- position-feedback (PPF). Results of the PPF simulations were compared with that of the LQR control and the advantage of using PPF control over LQR control in the finite element simulations is presented.
\end{abstract}

Keywords- active control; ANSYS; finite element model; linear quadratic regulator; LQR; piezoelectric actuators; positive position feedback; PPF

\section{INTRODUCTION}

The area of vibration analysis and control is an interesting and important field of research. In fact, any structure that has certain mass and elasticity is said to vibrate and so it is important to study the frequencies at which it vibrates to avoid resonance which can lead to failure of the system. The need to control such vibrations is important in the industrial field to get better functioning machines and increase the quality of the products. The importance of vibration control is also clear in the field of aerospace where flexible light weight structures are subjected to vibrations. Moreover, tall buildings and long bridges need vibration control to avoid their failure. In the past, passive vibration control was used in many structures by adding damping and stiffness but there were drawbacks for this type of control like slow response and increased weight of the structure. Therefore, active control techniques using piezoelectric materials attracted many engineers to be used in vibration control for their low weight and fast response. The use of piezoelectric materials as sensors and actuators is increasingly growing, as it can easily convert mechanical energy to electrical energy which is useful in many applications such as vibration control, energy harvesting and aerospace industry. Aldraihem [1] studied the effect of the location of the piezoelectric actuator on the response of the beam under different boundary conditions and formulated an optimization criterion based on the modal controllability. Al-Ashtari [2] derived a mathematical model presenting the smart cantilever beam and studying its behavior under different applied loads either static or cyclic. Moreover, he studied the effect of changing the number of actuators and proved that as the number of actuators increases, better damping of the oscillations is achieved.

For the field of vibration control using piezoelectric materials, Meyer et al. [3] studied the effect of PPF control and LQG control on flexible structures using piezoelectric materials. Then, Song et al. [4] proved that PPF control is robust to frequency variations and so it is an effective method for vibration suppression. Since ANSYS APDL (C) is more efficient in finite element modeling, researchers used to export the finite element matrices representing the model from finite element analysis software to Matlab ${ }^{\mathrm{TM}}$ and perform the control techniques. However, for complex structures it would be more efficient if the control algorithms can be implemented using the finite element software and withdrawing the need of exporting or importing the model. Malgaca and Karagulle [5] implemented a numerical model for a cantilever beam under free and forced response then integrated the control actions which are strain and displacement feedback with the finite element model using finite element program which is ANSYS APDL $\subset$. They also implemented the model experimentally and proved that numerical and experimental results are in good match. However, in all research mentioned there was no clear explanation of the method used to implement the controller using the finite element software until Takács and Rohal'-Ilkiv [6] implemented a digital LQR controller for a cantilever beam using ANSYS APDL $(\mathrm{C}$ and compared their results with the experimental results which were in a good match. This was the first implementation for a controller represented in state space form on finite element software, however only the LQR control was studied while other control techniques like PPF control are not yet implemented on the finite element software. The goal of this paper is to implement the positive position feedback (PPF) controller in ANSYS APDLC and compare ANSYS results with the PPF direct numerical integral of the closed loop system. Also, an optimal LQR controller is implemented and compared with that of PPF. 


\section{Piezoelectric CONSTITUTIVE RELATIONSHIPS}

In this study, piezoelectric materials are used in laminar configuration and so when positive voltage is applied, it bends up and when negative voltage is applied it bends down. For actuation purposes, the piezoelectric constitutive relationship can be represented as [7]:

$$
\begin{aligned}
& \sigma=C^{D} S-h D \\
& \epsilon=-h S+\beta^{S} D
\end{aligned}
$$

Where, $\sigma$ is the stress, $S$ is the strain, $C^{D}$ is the elastic coefficient measured under constant dielectric displacement $(\mathrm{D}=0), \in$ is the electric field, $\beta^{S}$ is the impermittivity constant measure under constant stress and $h$ is the piezoelectric constant which act similar to $d_{31}$ but for stress formulation of the constitutive relationship. So, we are concerned with $d_{31}$ mode [8] where the polarization direction is along the axis perpendicular to the plane in which the piezoelectric patch is bonded to the beam and according to the polarity of the applied field, the piezoelectric film expands or contracts in the transverse direction.

\section{MODELling OF A FiXED SMART BEAM}

The model considered in this study consists of an aluminum beam that is clamed at one end and a piezoelectric actuator bonded to it. Based on the Euler Bernoulli beam theory and the Hamilton's principle, the equation of motion of the model shown in Figure. 1 and Figure. 2 becomes [9]:

$$
\left.\rho(x) \frac{\partial^{2} w(x, t)}{\partial t^{2}}+\frac{\partial^{2}}{\partial x^{2}}\left(E I(x) \frac{\partial^{2} w(x, t)}{\partial x^{2}}\right)+B \frac{\partial w(x, t)}{\partial t}+C \frac{\partial^{2} w(x, t)}{\partial x \partial t}=M_{p o} G^{\prime \prime}(x)\right) V_{a}(t)
$$

Where $w(x, t)$ is the transverse displacement, $M_{p o}=-\frac{1}{2} * b^{*}\left(t_{b}+t_{p}\right) * E_{p} * d_{31}, E_{p}$ is the equivalent stiffness of the piezoelectric actuator, $d_{31}$ is the piezoelectric strain constant, $t_{b}$ and $t_{p}$ are the thickness of the beam and the piezoelectric patch respectively, $b$ is the width of the piezoelectric material and the beam, $\mathrm{B}$ is the coefficient of viscous damping, $\mathrm{C}$ is the coefficient of structural damping, $V_{a}(t)$ is the actuator voltage, $G^{\prime \prime}(x)=\frac{\partial^{2} G(x)}{\partial x^{2}}$ and $G(x)=H\left(x-l_{1}\right)-H\left(x-l_{2}\right)$ where $H(x)$ is the Heaviside function, $l_{1}$ and $l_{2}$ are the start and end locations of the piezoelectric material and the boundary conditions are: at $\mathrm{x}=0: \frac{\partial w(x, t)}{\partial t}=0$ and $w(x, t)=0$ while at $\mathrm{x}=\mathrm{L}: \frac{\partial^{2} w(x, t)}{\partial x^{2}}=0$ and $\frac{\partial}{\partial x}\left(\frac{\partial^{2} w(x, t)}{\partial x^{2}}\right)=0$

\section{Piezoelectric ActuAtor Model}

From the previous section, assuming the case of vibration without damping, Eq. 3 is simplified to be:

$$
\rho(x) \frac{\partial^{2} w(x, t)}{\partial t^{2}}+\frac{\partial^{2}}{\partial x^{2}}\left(\operatorname{EI}(x) \frac{\partial^{2} w(x, t)}{\partial x^{2}}\right)=M_{p o} G^{\prime \prime}(x) V_{a}(t)
$$

Which is a fourth order differential equation that represents together with the boundary conditions a boundary value problem for the smart cantilever beam. Knowing that the solution of Eq.4, which represents the general displacement of the beam at any time instant is separable, it can be represented as [9]:

$$
w(x, t)=\sum_{i=1}^{\infty} y_{i}(x) \eta_{i}(t)
$$

Where, $y_{i}(x)$ is the ith modeshape of the beam (spatial solution) and $\eta_{i}(t)$ is the generalized displacement (temporal solution). Using the orthonormality conditions and Eq.5, Eq.4 can be represented as:

$$
\omega_{r}^{2} \delta_{r s} \eta_{i}(t)+\delta_{r s} \frac{d^{2} \eta_{i}(t)}{d t^{2}}=M_{p 0} \int_{0}^{L} y_{i}(x) G^{\prime \prime}(x) d x V_{a}(t)
$$

And so by dividing the whole Eq.6 by $\delta_{r s}$, the equation of motion of the beam will be represented as:

$$
\ddot{\eta}_{i}(t)+\omega_{n i}^{2} \eta_{i}(t)=K_{a}\left[y_{i}^{\prime}\left(l_{2}\right)-y_{i}^{\prime}\left(l_{1}\right)\right] V_{a}(t)
$$

Where, $K_{a}=\frac{M_{p o}}{\delta_{r s}}$ and $\omega_{n i}$ is the natural frequency of the ith mode.

To include damping $\zeta_{i}$ in a second order system, Eq.7 becomes:

$$
\ddot{\eta}_{i}(t)+2 \zeta_{i}\left(\omega_{n i} \dot{\eta}_{i}(t)+\omega_{n i}^{2} \eta_{i}(t)=K_{a}\left[y_{i}^{\prime}\left(l_{2}\right)-y_{i}^{\prime}\left(l_{1}\right)\right] V_{a}(t)\right.
$$

Taking Laplace transform of Eq.8:

$$
s^{2} \eta_{i}(s)+2 \zeta_{i}\left(\omega_{n i} s \eta_{i}(s)+\omega_{n i}{ }^{2} \eta_{i}(s)=K_{a}\left[y_{i}^{\prime}\left(l_{2}\right)-y_{i}^{\prime}\left(l_{1}\right)\right] V_{a}(s)\right.
$$$$
\eta_{i}(s)=\sum_{i=1}^{\infty} \frac{w(x, s)}{y_{i}(x)}
$$

Using the relation $\eta_{i}(s)=\sum_{i=1}^{\infty} \frac{w(x, s)}{y_{i}(x)}$ from Eq.5, the transfer function relating the input voltage from the actuator to the output displacement of the beam will be:

$$
\frac{w(x, s)}{V_{a}(s)}=\sum_{i=1}^{\infty} \frac{K_{a} y_{i}(x)\left[y_{i}^{\prime}\left(l_{2}\right)-y_{i}^{\prime}\left(l_{1}\right)\right]}{s^{2}+2 \zeta_{i} \omega_{n i} s+\omega_{n i}^{2}}
$$

The transfer function presented in Eq.10 was used to perform the numerical simulations which were needed to validate the finite element model created using ANSYS and compare the results of the simulations with that of ANSYS. In this work only the first mode is included and so we have a second order system. However, if other modes are to be included then a higher order transfer function is obtained and same procedures followed but with more complex equations.

\section{MODELling USING ANSYS SOFTWARE}

ANSYS Mechanical APDLC is the finite element software used in this study. First, the model which consists of an aluminium beam with a piezoelectric material attached to it is 
created as shown in Figure. 1 and Figure. 2, and then the boundary conditions are specified. The properties of the aluminum beam and the piezoelectric material used are mentioned in Table 1 and Table 2 respectively.

\begin{tabular}{ll}
\multicolumn{2}{c}{$\begin{array}{c}\text { TABLE I .Dimensions and } \\
\text { properties of the beam }\end{array}$} \\
Dimension & Value \\
Length $[\mathrm{m}]$ & 0.37 \\
Width $[\mathrm{m}]$ & 0.03 \\
Height $[\mathrm{m}]$ & 0.001 \\
Density $\left[\mathrm{kg} / \mathrm{m}^{\wedge} 3\right.$ 3] & 2700 \\
Elastic modulus $\left[\mathrm{N} / \mathrm{m}^{\wedge} 2\right]$ & $69 \mathrm{e} 9$ \\
Moment of inertia $\left[\mathrm{m}^{\wedge} 4\right]$ & $2.5 \mathrm{e}-12$ \\
\hline
\end{tabular}

\begin{tabular}{|c|c|}
\hline \multicolumn{2}{|c|}{$\begin{array}{l}\text { TABLE II Dimensions and } \\
\text { properties of the piezoelectric } \\
\text { material from mide technology } \\
\text { [10]. }\end{array}$} \\
\hline Dimension & Value \\
\hline Length (m) & 0.0508 \\
\hline Width (m) & 0.03 \\
\hline Height (m) & $0.0381 \mathrm{e}-2$ \\
\hline $\begin{array}{l}\text { Elastic } \\
\text { modulus( }\left(\mathrm{N} / \mathrm{m}^{\wedge} 2\right)\end{array}$ & $6.7 \mathrm{e} 10$ \\
\hline
\end{tabular}

In ANSYS, the beam has been modeled using element solid 45 which include displacement degrees of freedom only while the piezoelectric material has been modeled using element solid 226 which includes electrical degree of freedom in addition to the displacement degrees of freedom. The two blocks of the aluminum beam and the piezoelectric material are created. Then, they were both meshed with element size of $5 \mathrm{~mm}$. After that, both blocks were bonded together using the contact manager of ANSYS APDL C. After creating the geometry, the boundary conditions were specified by setting the displacement degrees of freedom of the beam at the clamped end to zero. Coupling of all the nodes of the upper layer of the piezoelectric material was created to have the same control voltage while the nodes of the bottom layer have zero potential.

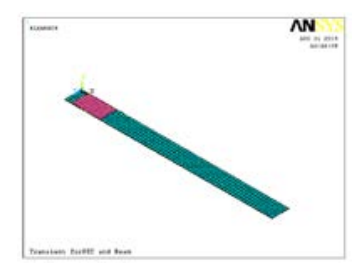

FIGURE I. MODEL USING ANSYS

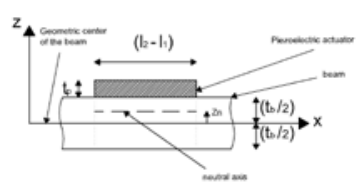

FIGURE II. Detailed View Of The Model
The damping used in this finite element analysis is Rayleigh damping, where the damping matrix $[C]$ is calculated using Eq.11.

$$
[C]=\alpha[M]+\beta[K]
$$

Where, $\alpha$ is the mass proportional Rayleigh damping coefficient and $\beta$ is the stiffness proportional Rayleigh damping coefficient. In this study, $\alpha$ is set to zero. Therefore, $\beta$ can be calculated from modal damping using Eq. 12 [11].

$$
\beta=\frac{2 * \zeta}{\omega}
$$

Where, $\zeta$ and $\omega$ are the modal damping and natural frequency of the highest mode included in the analysis which is the first mode in this study.

\section{Closed LoOP Simulation RESUlts}

The type of analysis used to implement the different types of controllers and plot the closed loop response in ANSYS APDL $($ is the transient analysis.

\section{A. Positive Position Feedback Control}

It was developed by Goh and Caughy to control vibrations of large space structures [12] then several researchers studied this control technique for its importance in different fields. In designing a controller usually a reduced order model is considered to make the mathematical model simpler to study. Therefore, some modes which were not considered in the reduced order model may be excited during operation which may lead to instability of the system which is known as the spillover effect. The main advantage of the PPF controller is its ability to overcome the spillover effect and to roll off at high frequencies thus ensuring robust stability of the system [13]. The PPF controller is designed by considering a single mode of the system which is represented by a second order system and design an auxiliary dynamic system representing the controller as in Eq.13 and Eq. 14, respectively [14].

$$
\begin{array}{r}
\ddot{x}+2 \zeta_{n} \omega_{n} \dot{x}+\omega_{n}{ }^{2} x=G \omega_{n}{ }^{2} \eta \\
\ddot{\eta}+2 \zeta_{c} \omega_{c} \dot{\eta}+\omega_{c}{ }^{2} \eta=\omega_{c}{ }^{2} x
\end{array}
$$

Where, $x$ is the modal coordinate of the system and $\eta$ is the modal coordinate of the controller, $\omega_{n}$ and $\omega_{c}$ are the natural frequencies of the structure and the controller, respectively and $\zeta_{n}$ and $\zeta_{c}$ are the damping coefficients of the structure and the controller, respectively. The closed loop system representing Eq. 13 and Eq. 14 is as shown in Figure. 3. 


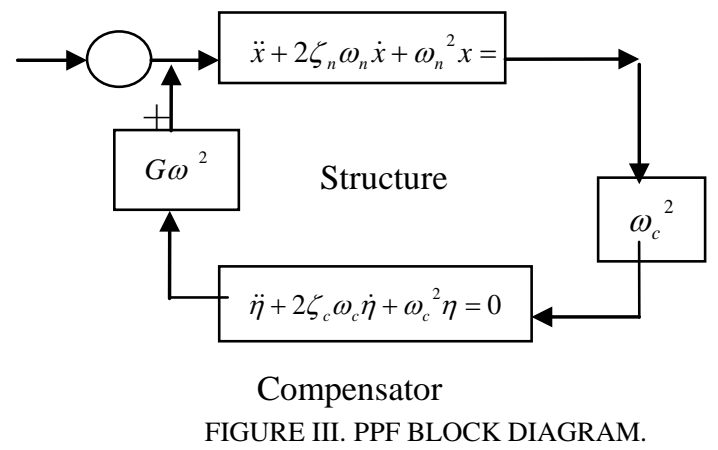

A discrete linear time invariant state space form of the system is needed to implement the controller using ANSYS APDL $\odot$. First the state space form of the first mode of the system is calculated at gain $\mathrm{G}=0.1$ and damping ratio $\zeta_{c}=1$, then using 'c2d' command in Matlab ${ }^{\mathrm{TM}}$, the discrete equivalent was found to be:

$$
A_{1}=\left[\begin{array}{cc}
0.9253 & -13.33 \\
0.009737 & 0.9325
\end{array}\right], B_{1}=\left[\begin{array}{c}
0.009737 \\
4.931 e-5
\end{array}\right] \text { and } C_{1}=\left[\begin{array}{ll}
0 & 1
\end{array}\right]
$$

While the discrete state space form of the compensator is found to be:

$$
A_{2}=\left[\begin{array}{cc}
0.4352 & -9.456 \\
0.006907 & 0.9463
\end{array}\right], B_{2}=\left[\begin{array}{l}
0.006907 \\
3.922 e-5
\end{array}\right] \text { and } C_{2}=\left[\begin{array}{ll}
0 & 1
\end{array}\right]
$$

The state space matrices are defined in ANSYS APDL $($ ) using *Dim command to define their size and *Set command to define its elements. The first step in the APDL code is to specify the type of analysis as mentioned at the beginning of this section. Then, the impulse force is applied at the free end of the beam. The main programming loop is defined using a do-loop:

$$
\begin{aligned}
& \text { *DO,t,2*Tstep,Tstop,Tstep } \\
& \text { *USE,ppfcon.mac } \\
& \text { TIME,t }
\end{aligned}
$$$$
\text { SOLVE }
$$

*ENDDO

Where *Use is a command used to call for another macro responsible for the control law implementation which acts as a subroutine.

$$
\text { *del,G,,nopr }
$$

*Dim,G,,2,1

*set,G(1,1),136.9,0 !G is the gain $\omega^{2}$
*MOPER,t1,A1,MULT,x1
*voper,u1,x2,mult,G
*VFACT,u1
*VFUN,t3,COPY,B1
*VOPER,x1,t1,ADD,t3

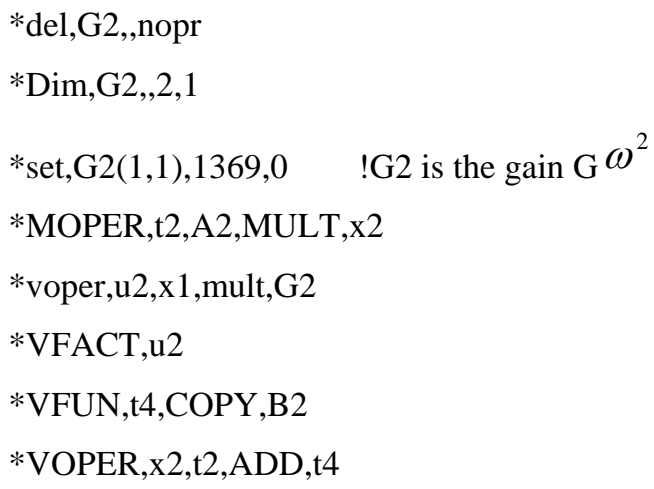

Where $\mathrm{G}$ and $\mathrm{G} 2$ are 2 vectors representing the two gains needed for the closed loop system which are $\omega_{c}{ }^{2}$ and $G \omega^{2}$. Finally the control signal is applied on the piezoelectric layer by selecting it first using NSEL command and the voltage is applied using d,all,volt,u. The resulting open loop and closed loop response is as shown in Figure. 4 and Figure. 5.

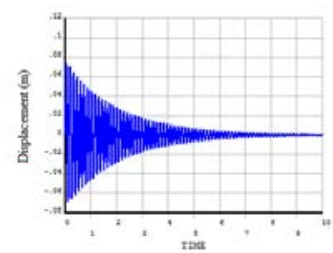

FIGURE IV. OPEN LOOP STATE SPACE RESPONSE.

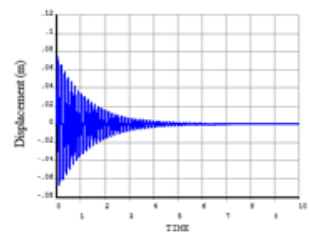

FIGURE V. CLOSED LOOP PPF RESPONSE.

From Figure. 4 and Figure. 5, it is noticed that the settling time is decreased from above $10 \mathrm{~s}$ to about $4 \mathrm{~s}$, this proves that the PPF technique is an efficient control method that can be implemented using ANSYS APDL ${ }^{\circ}$.

\section{B. LQR Control}

It is an optimal control algorithm that tries to achieve the desired performance while at the same time minimizing the control effort this is done by minimizing the performance index [15]. In order to use this type of control, a state space model is needed.

The control signal which represents the actuator voltage is calculated by Eq. 15 .

$$
u_{k}=-K x_{k}
$$

The control gain $\mathrm{K}$ is obtained using the 'dlqr' command in Matlab $^{\mathrm{TM}}$ : [K_lqr] =dlqr(A,B,Q,R). 
Where, $\mathrm{Q}$ and $\mathrm{R}$ are diagonal weight matrices for the state cost and the control cost respectively and their values in this

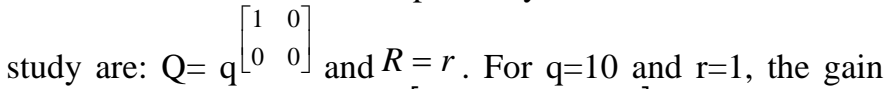
matrix is found to be: $\mathrm{K}=[2.4141-17.3305]$. For the model considered in this study the discrete state space matrices are obtained using 'c2d' command in Matlab ${ }^{\mathrm{TM}}$ and where found to be:

$$
\mathrm{A}=\left[\begin{array}{cc}
0.9235 & -13.6695 \\
0.0097 & 0.9308
\end{array}\right], \mathrm{B}=\left[\begin{array}{c}
0.0097 \\
0
\end{array}\right] \text { and } \mathrm{C}=\left[\begin{array}{ll}
0 & 1
\end{array}\right] \text {. }
$$

The APDL code for the implementation of the LQR controller is the same as that of the PPF but the control law is changed to be:

$$
\begin{aligned}
& * \text { MOPER,ut,K,MULT, } \mathrm{x} \\
& \mathrm{u}=\text {-ut(1,1) }
\end{aligned}
$$

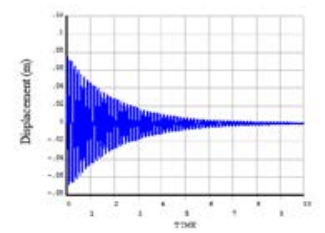

FIGURE VI. OPEN LOOP STATE SPACE RESPONSE.

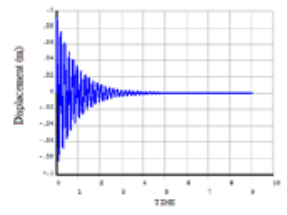

FIGURE VII. CLOSED LOOP LQR RESPONSE AT Q=10 AND R=1.

As shown in Figure. 6 and Figure. 7, the closed loop response at $\mathrm{q}=10$ and $\mathrm{r}=1$ reaches steady state in about $4 \mathrm{~s}$ which is nearly similar to that of the PPF control.

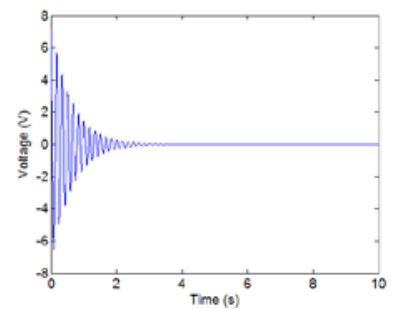

FIGURE VIII. LQR CONTROL VOLTAGE.

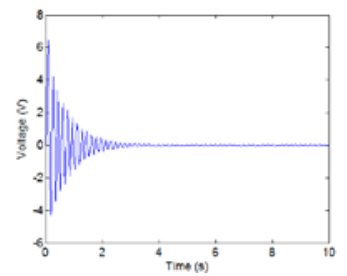

FIGURE IX. PPF CONTROL VOLTAGE.
From Figure. 8 and Figure. 9, the control voltage required to achieve the desired steady state response in both control techniques is $6 \mathrm{~V}$. This proves that LQR is an efficient control method as well as the PPF control as they both reach the desired response with low control effort. However, what makes the PPF control better than the LQR is the way of implementation in finite element softwares as LQR control requires using Matlab $^{\mathrm{TM}}$ to calculate the gain $\mathrm{K}$ discussed before while PPF control can be implemented entirely using the finite element software without the aid of any other softwares.

\section{CONCLUSION}

In this paper, digital PPF and LQR controllers were implemented in ANSYS APDL $\odot$ software. It is proved in this work that modern control techniques using state space forms can be integrated in the finite element software instead of exporting the finite element model and importing it to a simulation environment as Matlab ${ }^{\mathrm{TM}}$ to apply the control law. By comparing the finite element results of the open loop and closed loop response of the two techniques with the numerical results, they were in good match. Also it was found that both PPF and LQR control techniques are efficient as they can achieve the desired response at low control voltage. However, it was found that the implementation of the PPF controller using ANSYS is easier than the LQR technique as it can be implemented entirely using finite element software without the need to export the finite element model to any other software.

\section{REFERENCES}

[1] O.J. Aldraihem, R. Singh and R. C. Wetherhold, Optimal Size and Location of Piezoelectric Actuator/Sensors: Practical Considerations, Journal of Guidance, Control and Dynamics, Vol. 23, No. 3, 2000.

[2] W.K. Al-Ashtari, The Deflection Control of a Thin Cantilever Beam by Using a Piezoelectric Actuator / Sensor, The 1stRegional Conference of Eng. Sci. NUCEJ Spatial Issue vol.11,No.1, 2008, 43-51.

[3] J. L. Meyer, W. B Harrington, B. N. Agrawal and G. Song, Vibration suppression of a spacecraft flexible appendage using smart material, Smart Mater. Struct. 7, 1998, 95-104.

[4] G. Song, S. P. Schmidt and B. N. Agrawal, Experimental study of vibration suppression of flexible structure using modular control patch, J. GUIDANCE, vol. 25, No. 1, 1998.

[5] H. Karagulle, L. Malgaca, Numerical and experimental study on integration of control actions into the finite element solutions in smart structures, Shock and Vibration 16, 2009, 401-415.

[6] G. Takács, B. Rohal-Ilkiv, ” Modeling active vibration control systems through finite element analysis software", The 4th International conference - Modeling Of Mechanical And Mechatronic Systems, Faculty of Mechanical engineering, Technical university of Košice, Slovak, 2011.

[7] N. Jalili, Piezoeelctric-Based Vibration Control, Springer Science+Business Media, New York, 2010.

[8] Q.Q. Zhang, S. J. Gross, S. Tadigadapa, T. N. Jackson, F. T. Djuth and S. Trolier-Mckinstry, Lead zirconate titanate films for d33 mode cantilever actuators, Sensors and Actuators A: Physical 105, 2003, 91-97.

[9] L. Meirovitch, Fundamentals of Vibrations,McGraw-Hill, New York, 2001.

[10] Mide, Engineering Smart Technology Products on http://www.mide.com/.

[11] Damping, Mechanical APDL theory, Release 14.0, SAS IP, Inc.

[12] C. J. Goh, T. K. Caughy, On stability problem caused by finite actuator dynamics in the collocated control of large space structures, International Journal of control 41, 1985. 
[13] C. Spier, I. S. Sadek, J. C. Bruch and J. M. Sloss, Active Displacement Feedback Control of a Smart Beam: Analytic and Numerical Solutions, Proceedings of the International MultiConference of Engineers and Computer Scientists II, 2009.

[14] D. Inman, Vibration with control, John Wiley \& Sons Ltd, England, 2006.

[15] K .Ogata, Modern Control Engineering, 5th edition, Pearson Education, Inc., 2010. 